\title{
La opacidad de la oralidad en el aula de clase: un reto en la docencia
}

\section{The opacity of the orality in the classroom: a challenge in teaching}

\author{
Sandra Patricia Tafur-Muñoz \\ patriciatafurm1@gmail.com \\ Universidad Distrital Francisco José de Caldas, \\ Colombia \\ Nelsy Jhoanní Armesto-Argüelles \\ nelsyarmesto@gmail.com \\ Universidad Distrital Francisco José de Caldas, \\ Colombia
}

Tafur-Muñoz, S. P. \& Armesto-Argüelles, N. J. (2019). La opacidad de la oralidad en el aula de clase: un reto en la docencia. Oralidad-es, 5, 1-9. https://revistaoralidad-es.com/index.php/ro-es/article/view/115/84

Fecha de recepción: 09 de agosto de 2019 - Fecha de aceptación: 02 de noviembre de 2019 


\section{Resumen}

El desarrollo de la oralidad en el aula de clase es una de las realidades que enfrenta el docente investigador, es por ello que este artículo de reflexión reconoce que, desde la perspectiva lingüístico-discursiva, el orden pedagógico e investigativo puede comprender y resignificar la importancia de la oralidad en el proceso formativo del estudiante. Esta propuesta se enfoca desde la investigación pedagógica de las actividades discursivas de la lengua y el análisis crítico del discurso (ACD) a fin de teorizar y generar dinámicas que, a corto y largo plazo, reduzcan las asimetrías sociales a través del lenguaje al tiempo que se disminuyen los niveles de glosofobia resultado de las relaciones de poder en el aula.

Palabras clave

Oralidad; discurso; glosofobia; relaciones de poder

\section{Abstract}

The development of oral skills in the classroom is one of the realities the researcher teacher deals with, that is the reason due to this Reflection article recognizes that from the linguistic - discursive perspective, the pedagogic and research order can understand and re- signify the importance of oral skills in the formative process of students. This proposal is focused on the Language Discursive Activities Pedagogic Research and on The Critical Discourse Analysis (ACD) to provide theoretical accounts and generate dynamics which, in short and long term, reduce social asymmetries through language and at the same time diminish the levels of glossphobia resulting from power relations in the classroom.

Keywords

Orality; speech; glossophobia; power relationships 


\section{Introducción}

El lenguaje ha cumplido un papel fundamental en la formación de la humanidad y en la creación de individuos pensantes capaces de representar o mostrar, por medio de él, su mundo real y a la vez concebir la variedad de mundos que sus pares o demás perciben. Cárdenas (2013) define el lenguaje como un "fenómeno de sentido abierto que ocurre en contextos de alteridad definidos, tiene apoyo semiótico y teje redes de enunciados interdiscursivos, en situaciones sociales definidas" (Cárdenas, 2013, p.315). Por consiguiente, el lenguaje se podría considerar como el medio fundamental en la interacción humana ya que permite establecer relaciones de comunicación con indefinidos actores y en multiplicidad de escenarios.

Por su parte, los usos del lenguaje en relación con las asimetrías modelan pautas políticas, sociales, educativas y sin lugar a dudas discursivas, las cuales son capaces de determinar las habilidades comunicativas de un ser humano en un contexto específico. Así mismo y de acuerdo con Van Dijk (2003), la producción y la reproducción se asocian con el poder directamente en el discurso, visto así la desigualdad social también se hace presente en la comunicación y el uso de la lengua, marcando así evidentes asimetrías sociales. En concordancia con lo anterior, se podría determinar que el margen de diferencia entre los usos del lenguaje está determinado por factores como el consumo de cultura, el nivel académico y el acceso a información entre otros.

Dentro de estas prácticas es necesario remitirse a la oralidad, por ello vale la pena aclarar que esta debe ser entendida no solo como el acto humano de hablar, sino como un sistema de signos que acompaña el discurso, así se determina en el estudio presentado por los investigadores Soler \& Baquero (2014) "[...] los discursos no son sólo palabras, sino palabras "acerca de algo" y, sobre todo, palabras con las que "hacemos algo" (Soler $\&$ Baquero, 2014, p.15). Por lo anterior se puede afirmar que las palabras tienen poder y no sólo en su significado sino en el acto comunicativo dando así un enorme valor a la oralidad como acto discursivo.

Ahora bien, se quiere tomar como punto de partida y base para la temática, la experiencia docente, la cual inicia desde la práctica pedagógica, la investigación curricular e incluso las propias vivencias en el colegio y en la universidad, donde se consideran las falencias que los estudiantes en general, poseen a la hora de interactuar con sus pares o de desenvolverse ante un público. No se trata aquí de buscar culpables o victimarios, simplemente con esto se ven varios aspectos a tratar respecto a la oralidad ya que en la mayoría de los casos el papel que cumplen los docentes titulares es fundamental, bien sea para mitigar o desarrollar la competencia comunicativa oral.

Sin embargo, el rechazo que los estudiantes tienen a hablar está ligado al discurso que realizan los docentes así que cuando se produce un choque entre el discurso y el contexto ocurre la "glosofobia", la cual es definida por el Gabinete psicopedagógico de la Universidad de Granada como la sensación de miedo al hablar en público: "Estas situaciones suelen ir acompañadas de diversos miedos, como a ser evaluado negativamente por los demás, a no ser escuchados, a no tener nada interesante que decir, y/o a la vergüenza que se pueda pasar frente a un posible fracaso" (UGR, 2005, p. 1), por ello es necesario entender que el poder y el abuso de poder visto en todas las esferas sociales permite analizar cómo la superioridad en determinadas situaciones restringe o limita la interacción ya que cuando se pone de manifiesto la jerarquía, los factores psicosociales motivan o restringen la oralidad.

Luego de revisar los anteriores postulados, la práctica pedagógica, las vivencias y la relación que directa e implícitamente se genera entre lenguaje, interlocutores, poder y discurso, se produce el cuestionamiento que conducirá al 
desarrollo de esta reflexión sobre el lugar de la oralidad en el aula, ¿Cómo desarrollar la oralidad en el aula para disminuir las asimetrías sociales a fin de reconocer la oralidad en los estudiantes desmitificando el trato diferencial recibido por parte de sus docentes?

Para atender a lo anterior y a la pregunta formulada, en este artículo se hará referencia sobre los factores que han dado paso a la invisibilización de la expresión oral (glosofobia) en el aula; luego se analizarán las asimetrías sociales y relaciones discursivas de poder que discriminan y controlan formando una desigualdad entre el docente y el estudiante. Todo ello para fundamentar teóricamente la importancia que tiene la expresión oral en el sujeto, sobre todo en el aula, y su contexto; y por último se considerará una propuesta que vincule la investigación en el aula para que el estudiante adquiera niveles de pronunciación y nuevo léxico que pueda utilizar sin temor a la censura.

\section{Desarrollo}

\section{Perspectiva lingüístico discursiva}

Para considerar los factores que han dado paso a la glosofobia en el aula, se debe considerar que el discurso al ser mediado y ejecutado en la oralidad da cuenta de que el lenguaje se encuentra presente en el hombre por medio de tres ramas: el lenguaje de la acción que hace referencia a la cultura oral; el lenguaje de las ideas que hace alusión a la cultura escrita y el lenguaje de la imagen que hace mención a la cultura virtual. Estas tres vertientes del lenguaje, propuestas por Gutiérrez-Ríos (2014), muestran a su vez, el papel que tiene la oralidad y el discurso en las interacciones humanas y en el lenguaje mismo, pues como es notorio, por medio de la oralidad se le otorga sentido a la capacidad de contacto que genera la interacción oral y se experimenta un encantamiento al estar cara a cara despertando mayor actividad sensorial con el otro que nos escucha y nos muestra igualmente un punto de vista.

No obstante, estas vertientes del lenguaje se complementan con los rasgos retóricos, enunciativos y lingüísticos de la lengua oral, incluyendo sus funcionamientos y clasificación de sus textos y discursos, es por ello que se parte de la dimensión lingüístico-discursiva, donde el discurso oral, se concibe como un ámbito discursivo que define, regula y analiza los textos orales. De igual manera, la palabra hablada en el aula, se define en el campo pedagógico y se encuentra mediada por la interacción dialógica existente entre quienes aprenden y enseñan en el sistema escolar. La lengua oral se ve aquí en la organización del ambiente de aprendizaje, la explicación de los contenidos y la comprobación de si éstos se han asimilado correctamente.

Sin embargo, cuando se restringe el uso oral en el aula ocurre una invisibilización a la capacidad discursiva, a las interacciones humanas y a las vertientes del lenguaje. Esto evita que se quiera distinguir la oralidad que pertenece a los estudiantes y, en sí mismo, esto es un problema ya que un factor que aumenta la glosofobia es no escuchar la voz de los jóvenes, pues como docentes son los educandos el foco de atención y el aula de clase el pretexto para desarrollar e incentivar esa aptitud que favorece al desarrollo personal y la construcción de identidad social. Además, es por medio de la oralidad que los alumnos integran nuevos conocimientos, procesan informaciones verbales, aprenden a comunicar, dominar los géneros orales y códigos de civilidad escolar y social.

De acuerdo con lo anterior, es claro que existe una dificultad discursiva oral en los educandos y el orden pedagógico que plantean los docentes, esto permite afirmar que una intervención en aula sería pertinente a fin de que los estudiantes, con el apoyo de los docentes, logren evitar o dis- 
minuir la glosofobia dentro y fuera del aula. Es por ello que en la actualidad la preocupación por la competencia comunicativa oral ha crecido, razón por la cual se ha desarrollado esta reflexión desde la línea de investigación propuesta por la Maestría en Pedagogía de la Lengua Materna de la Universidad Distrital Francisco José de Caldas, con ello se ha relacionado la línea Pedagogía de las actividades discursivas de la lengua ya que "esta línea se centra en la investigación sobre las modalidades del lenguaje (oralidad y escritura) y sus actividades específicas: hablar, escuchar, leer y escribir en diversos ámbitos de la acción social, expresadas a partir de diversos tipos de discursos y textos." (p1). Para el caso específico de este artículo nos centraremos en la modalidad oral del lenguaje y su actividad específica, hablar, dado que esta habilidad se encuentra estrechamente vinculada con el discurso, lo cual permite investigar sobre el lugar que otorga esta modalidad a la relación de producción discursiva por parte del estudiante y el docente.

Lo anterior se hace esencial para este escrito luego que enmarca la orientación que desde la universidad conduce a las investigadoras hacia la oralidad, desglosando los diferentes campos específicos que esta actividad discursiva desarrolla. Además, brinda un acompañamiento desde el campo académico, dando así mayor solidez a esta investigación, para que se logre comprender y resignificar la importancia de la oralidad en el proceso formativo del estudiante.

\section{Orden pedagógico}

Desde el campo teórico se evidencia que sobre la oralidad, Soler (2012), propone el ACD (Análisis crítico del discurso) como una herramienta esencial para la comprensión de los discursos de y sobre la escuela. Asimismo, la capacidad discursiva se relaciona "con el poder, el abuso de poder y con la producción y reproducción de estos en el discurso" (Soler, 2012, p.133). Al utili- zar el ACD como herramienta para comprender el discurso de los docentes y los estudiantes en el aula podrá realizarse una transformación en pro de las actividades discursivas de la lengua. Es así que para poder resolver la pregunta formulada los docentes deben orientarse en un proyecto que posibilite transformar las prácticas discursivas asociadas a la oralidad en nuestro contexto educativo a partir de la observación participante y el papel del docente en inter-relación con el estudiante.

Todo lo anterior permite que el docente reconstruya su práctica, "el docente dejará de ser un técnico para ser un profesional autónomo, que se responsabiliza por sus acciones, reflexiona sobre su práctica conjuntamente con sus pares y está dispuesto a cambiar para mejorarla. [...] Será aquel que somete a consideración hipótesis de trabajo, reflexiona sistemáticamente sobre su praxis, comunica y delibera con sus colegas sobre lo realizado y lo logrado" (Rodríguez, 2007, p.10). De esta manera la profesión docente se orienta sobre las investigaciones que reflexionen y fundamenten la oralidad y la escritura en los diferentes contextos de aprendizaje junto con sus actividades particulares en una perspectiva dialógica e interaccionista, ya que "tenemos que avanzar en el conocimiento de cómo puede contribuir la enseñanza al desarrollo de las capacidades y las habilidades verbales de los niños $\mathrm{y}$ adolescentes de manera integrada, de forma que se desarrollen armónica y profundamente" (Camps, 2012, p.35). Así, se puede concluir que el docente tiene la obligación de actualizar sus saberes, a fin de construir sus intervenciones de clase basado de la reflexión y la reestructuración del saber.

Así pues, el trabajo investigativo de las actividades discursivas permite que el proyecto no se oriente a buscar culpables o victimarios, sino que se profundice en indagar sobre los factores que inciden en el discurso oral de los estudiantes, ya que en la mayoría de los casos el papel que cumplen los docentes es fundamental bien sea 
para mitigar las asimetrías sociales o para desarrollarlas a la hora de construir conocimientos usando la oralidad, pues es en la reflexión docente que se puede hablar de saber pedagógico, así pues "la crítica de pares profesionales, es decir, de otros maestros investigadores, y la autocrítica de la práctica pedagógica, han permitido un desarrollo profesional que ha llevado a procesos liberadores de los maestros investigadores." (Restrepo, 2004, p.54). Para ello, es importante tener una claridad sobre el papel del docente como investigador en el aula, pues de acuerdo con Cerda (2007) los maestros se transforman en objetos y sujetos de investigación, esto quiere decir que para ahondar en la oralidad de los estudiantes y los factores que de ella se desprendan se debe partir de una investigación pedagógica que permita realizar aportes en el campo educativo.

\section{Hacia una investigación en el aula}

En cuanto a investigación se refiere, son significativos los avances, día tras día los docentes de los diferentes grados de escolaridad hacen presencia con intervenciones ya sea en el aula o de mayor impacto, institucionales, regionales, nacionales, etc. Históricamente las universidades, en especial los programas de posgrado o maestría han tenido la responsabilidad implícita de favorecer el entorno investigativo y en respuesta a esto, preparan estudiantes con un enfoque global que les permite ver no sólo las necesidades que se generan en su entorno sino las necesidades en un campo amplio aportando soluciones de gran efectividad. Sin embargo, ha hecho falta que los docentes entiendan la necesidad de investigar en el aula, más aún, cuando se presentan problemáticas como la glosofobia.

Es por ello que en el campo de una investigación que permita la escucha de la voz del estudiante y la actualización docente se presenta una bifur- cación (La investigación propiamente dicha y La investigación formativa). Para efecto de esta publicación, la primera se verá tal como la define Hernández (2002) "La investigación propiamente dicha es una empresa internacional. Cuando se habla de la investigación propiamente dicha se alude necesariamente a la existencia de grupos consolidados de investigación que producen resultados (artículos, libros, patentes, innovaciones tecnológicas de diverso tipo) reconocidos más allá de las fronteras nacionales, de grupos que están en contacto permanente, en intercambio permanente con las comunidades académicas nacionales e internacionales" (p.82) Así pues, la investigación propiamente dicha permite a los grupos, tener reconocimiento, mientras establecen relaciones con pares a fin de debatir posturas $\mathrm{y}$ creencias, mientras se incrementa el conocimiento.

Por su parte la investigación formativa es en sí misma es una actividad pedagógica propia de los docentes- investigadores que "realizan el contacto directo con los estudiantes, ya sea como actividad de aula, tesis de grado, o monografía, aplicación de saberes alrededor de un objeto de estudio o realización de talleres de innovación para cualificar los procesos" (Cerda, 2007, p.10) Para un docente debe resultar importante vincular la noción de investigación formativa porque al trabajar con conocimientos pedagógicos ya validados por los estudiantes o el ámbito educativo como lo es la existencia de la oralidad, ocurrirá una transformación en el aula, pero será labor del docente realizar un ejercicio pedagógico que posibilite resolver la pregunta planteada en aras de profundizar y transformar la educación en forma positiva, pues "no basta saber de pedagogía para ser exitoso en la educación, (...)En este sentido, el saber pedagógico es la adaptación de la teoría pedagógica a la actuación profesional, de acuerdo con las circunstancias particulares de la personalidad del docente y del medio en el que debe actuar." (Restrepo, 2004, p.47). De acuerdo con lo anterior, la investigación formativa, 
conduce al docente no sólo a la capacitación y el crecimiento académico sino a la transposición didáctica esperando obtener como resultado un verdadero proceso de enseñanza - aprendizaje.

Es de destacar, que al relacionar la noción de oralidad en la escuela con la noción de investigación formativa se pueden determinar métodos flexibles que se adecúen a las necesidades y exigencias que surjan durante el proceso de investigación que demande un proyecto educativo. Ahora bien, para esta propuesta reflexiva es necesaria la relevancia que se da a la experiencia del sujeto puesto que en la investigación formativa (Cerda, 2007) el docente transforma su propia experiencia y se respalda por las experiencias que tienen los estudiantes, esto da cuenta de un criterio de validez determinado por los efectos y resultados a nivel pedagógico.

Al pensar en esta investigación, enfocada hacia determinar de qué manera el lenguaje puede disminuir las asimetrías sociales a fin de reconocer la oralidad en los estudiantes, resulta pertinente reconocer un vínculo con la investigación educativa, porque la acción oral y su propia investigación se dará en las aulas para así conceptualizar, por ejemplo, la noción que los estudiantes tengan de oralidad, es decir, que ellos como participantes de este proyecto den cuenta de su perspectiva sobre la misma. Además, podrá ser apoyada con estudios de caso, así como la participación de docentes, pues la escuela y sus integrantes son los encargados de potencializar las habilidades y desarrollar las capacidades del estudiante. Es justo allí, donde la investigación educativa adquiere mayor relevancia ya que se centra en la acción en clase mientras son revisados los estudios de caso, resignificando conceptos conforme se estudian los mismos.

Esta propuesta se enfoca desde la investigación pedagógica de las actividades discursivas de la lengua y el análisis crítico del discurso (ACD), ya que resultado de la recolección de datos con estudiantes de ciclo $\mathrm{V}$ de dos colegios distritales de Bogotá se ha logrado evidenciar que la opacidad de la oralidad, se deriva, entre otras cosas de la evidente dominancia que del discurso tiene el docente al interior del aula. Esta propuesta busca mejorar la competencia comunicativa oral para transformar la voz silente del estudiante a través del debate. Esto será posible a través del diseño e implementación de una propuesta pedagógica que permita resignificar los procesos de habla desde las actividades pedagógicas y didácticas que permitan el desarrollo de habilidades de la competencia comunicativa oral disminuyendo las asimetrías sociales dentro y fuera del aula.

En cuanto a la disminución de las asimetrías sociales, se espera que a corto plazo los estudiantes vayan ganando seguridad y apropiación de las habilidades discursivas orales, así se atreverán a hacer intervenciones en el aula y no sólo de una manera formal o mediada por el docente, sino de manera voluntaria, lejos del temor al veto por parte del profesor. Lo anterior como resultado de la implementación de talleres en los que se potencie la oralidad. A largo plazo se espera que los estudiantes logren un completo desarrollo de su competencia comunicativa oral, que consigan a través de las palabras comunicar, exponer, debatir y dialogar, tanto en un ámbito formal como en uno informal, evidenciando así la disminución de la glosofobia.

\section{Conclusiones}

El solo hecho de que el docente participe en el aula y de ella surja una transformación de su experiencia para generar la construcción de conocimientos permitirá identificar y resignificar su rol como docente investigador pues lograr una relación dialógica entre la teoría pedagógica y los saberes pedagógicos permitirá lo que Chevallard (1988) denomina como una adecuada "transposición didáctica" que consiste en "la transformación del saber científico a un saber posible de ser enseñado” (Cerda, 2007, 
p.54). Esta transposición es necesaria para que el docente reconozca la oralidad del estudiante y la motive porque sólo cuando el docente re-evalúa su práctica, la reconstruye y la evalúa en pro del mejoramiento continuo genera un efecto social sobre el lenguaje y para este caso específico conduce a la reducción de las asimetrías sociales reconociendo la importancia en el desarrollo de la oralidad en los estudiantes y la disminución del miedo al decir, nombrado a lo largo de este texto como glosofobia.

En definitiva, este proceso reflexivo de oralidad, busca teorizar y generar dinámicas que a corto y largo, plazo reduzcan las asimetrías sociales a través del lenguaje. Por otra parte se espera disminuir los niveles de glosofobia resultado de las relaciones de poder en el aula. Con lo anterior, se espera dar cuenta de una transformación didáctica al interior del aula aunque con impacto dentro y fuera de ella.

Después de este primer recorrido frente a las asimetrías sociales y los problemas de oralidad que pueden generarse a causa de éstas, lo que queda por decir es que conforme la sociedad avanza van apareciendo nuevos sentidos culturales que se enmarcan en la escuela, así que la labor docente tendrá como reto generar transformación en las manifestaciones discursivas de tal manera que se redefina el actuar del profesorado. A partir de esto es que se puede alimentar el proceso individual del estudiante hacia el diálogo de experiencias para mantener una interacción y comunicación en diversas visiones de mundo y romper las prácticas pedagógicas tradicionales que deslegitiman el proceso oral del estudiante.

\section{Referencias}

Camps, A. (2012). "La investigación en didáctica de la lengua en la encrucijada de muchos caminos". Revista Iberoamericana de Educación. N. ${ }^{\circ} 59$, p 35

Cárdenas, P. (2013). Campo del lenguaje y la formación. En: Cátedra doctoral: campo intelectual de la educación y la pedagogía. $\mathrm{N}^{\circ} 1$, 313-335, Editores: Germán Vargas Guillén, alexander Ruiz Silva, Doctorado Interinstitucional en Educación- DIE, Universidad Pedagógica Nacional.

Cerda, H. (2007). La pedagogía como investigación. Cap.1 Investigación y enseñanza una relación controvertida. Cap. 2. Conocimiento científico $y$ saber pedagógico. Cap. 3. Investigación en strictu sensu e investigación formativa (p.15-68). Cooperativa Editorial Magisterio. Bogotá.

Chevallard, Yves (1998), “Qué I es la transposición didáctica?”, “Existe 1 la transposición didáctica? O la vigilan«ia epistemológica”, "buena o mala la transposición didáctica?" y "Objetos de saber' $y$ otros objetos", en La transposición didáctica. Del saber sabio al saber enseñado, Claudia Gilman (trad.), ed” Argentina, Aique (Psicología cognitiva y educación), pp. 45-66.

Gutiérrez-Ríos, M. Y. (2014). Concepciones y prácticas sobre la oralidad en la educación media colombiana. Tesis Doctoral. Universidad Distrital Francisco José de Caldas. Bogotá D.C. Recuperado de http://die.udistrital.edu.co/sites/ default/files/doctorado_ud/publicaciones/ concepciones_y_practicas_sobre_la_oralidad en_la_educacion_media_colombiana.pdf

Hernández, C. (2002). Universidad y Excelencia En: Educación Superior Sociedad e Investigación. Cuatro Estudios Básicos sobre Educación Superior. Bogotá: Colciencias - Ascun. 
MAESTRÍA EN PEDAGOGÍA DE LA LENGUA MATERNA. Línea de investigación Pedagogía de las actividades discursivas de la lengua. Actividades discursivas de la lengua (p.13) ANEXO 12

Restrepo, B. (2004). La investigación-acción educativa y la construcción de saber pedagógico. Educación y educadores. Recuperado de http://educacionyeducadores.unisabana.edu. co/index.php/eye/article/view/548/641

Rodríguez, N (2007) Los tres paradigmas de la investigación en educación. Universidad Central de Venezuela. Disponible en http:// saber.ucv.ve/bitstream/123456789/9671/1/ Los\%20Tres\%20Paradigmas.pdf

Soler, S. (2012). Análisis crítico del discurso y educación. Una interrelación necesaria. Universidad Distrital Francisco José de Caldas. Bogotá, Colombia - Agosto de 2012. Recuperado de https://es.scribd.com/document/275051381/ Analisis-critico-del-discurso-y-Educacion-Soler-Castillo-2012-pdf

Soler, S. \& Baquero, P. (2014) Maestros, hegemonía y contra discurso (PP. 15, 16). Universidad Distrital Francisco José de Caldas

Van Dijk, T. (2003) La multidisciplinaridad del análisis crítico del discurso: un alegato en favor de la diversidad. Métodos de análisis crítico del discurso. Ruth Wodak \& Michael Meyer, Barcelona: Gedisa, pp. 143-177.

UGR, Gabinete psicopedagógico (2005). Ansiedad a hablar en público. Consultado en: http://www.ugr.es/ cbustos/APOYO\%20 DOCENTE/ORIENTACION/enlaces/docu$\underline{\text { mentos/ansiedad-hablar.pdf }}$ 